\title{
Using Qualitative Analysis to Assess a Model of Support for Online Health Communities for People Living with Chronic Health Conditions
}

\author{
Brian M Green ${ }^{*}$ Kaitlyn Van Horn ${ }^{1 *}$; Ketki Gupta ${ }^{1 *}$; Amrita Bhowmick ${ }^{1 *}$; Michael Booth ${ }^{2 *}$ \\ ${ }^{1}$ Health Union, LLC, Philadelphia, PA, United States \\ ${ }^{2}$ School of Public Health, University of Michigan, Ann Arbor, MI, United States \\ *all authors contributed equally
}

Corresponding Author:

Brian M Green

\begin{abstract}
Background: Online health communities $(\mathrm{OHC})$ can be a powerful tool to facilitate communication among patients, professionals and family members who live with or care for someone with a chronic health condition(s). Health Union LLC's OHC model engages, empowers and encourages people to take an active role in their health by providing content that aligns with their needs and interests and by cultivating a safe environment where communication, understanding and meaningful relationships can thrive. OHCs included in this study target people living with multiple sclerosis, migraine, IBS, rheumatoid arthritis, lung cancer, and prostate cancer.
\end{abstract}

Objective: Using qualitative methods we sought to determine if constructs in the Health Union OHC model are supported by themes identified in OHC participant comments. Key components of the model to be tested include: content tailored to needs of community, facilitation, and encouragement of social support, active moderation, opportunities for active and passive engagement, and transparency of community norms and rules.

Methods: A sample of over 5800 comments exported from over 40 Facebook posts from 6 OHCs was analyzed using the Dedoose qualitative data analysis software. Comments from these Facebook posts were extracted, imported into Dedoose software and coded. Interrater reliability of initial coding was calculated using Pearson Correlation Coefficient. An exploratory approach was taken in the analysis and initial codes were grouped into thematic categories and then confirmed through thematic network/framework analysis using the Dedosse software tool. Thematic categories were compared for similarity and differences for each of the $6 \mathrm{OHCs}$, original post type, and by the extent of active moderation evident in each comment thread.

Results: Qualitative thematic network analysis of posts and comments from 6 OHCs correspond to the primary components of the Health Union OHC model. This analysis suggests that the structural elements of the OHC model, including active site moderation, support high levels of community engagement and information sharing and mutual support of OHC participants.

Conclusions: Qualitative data from the 6 OHCs demonstrates the positive impact the community has on participants, often helping them reframe their health care experience and coping strategies. The principle of adaptive engagement is demonstrated by the thematic network analysis and illustrates the Health Union OHC model constructs. Different community segments have different patterns of engagement. Our primary focus on the content of participant comments in this analysis is a current limitation. While we also examine more passive methods of liking and sharing posts utilized by OHC participants, these may warrant further analysis. This study has practical significance as it helps to demonstrate the value of online health communities for people living with chronic health conditions by providing meaningful engagement, support, and information in an accessible environment.

(iproc 2018;4(2):e11774) doi: 10.2196/11774

\section{KEYWORDS}

online patient communities; qualitative research; social media; social sharing; social support 
Edited by T Hale; this is a non-peer-reviewed article. Submitted 01.08.18; accepted 29.08.18; published 17.09.18.

Please cite as:

Green BM, Van Horn K, Gupta K, Bhowmick A, Booth $M$

Using Qualitative Analysis to Assess a Model of Support for Online Health Communities for People Living with Chronic Health

Conditions

iproc 2018;4(2):e11774

URL: http://www.iproc.org/2018/2/e11774/

doi: $10.2196 / 11774$

PMID:

CBrian M Green, Kaitlyn Van Horn, Ketki Gupta, Amrita Bhowmick, Michael Booth. Originally published in Iproceedings (http://www.iproc.org), 17.09.2018. This is an open-access article distributed under the terms of the Creative Commons Attribution License (https://creativecommons.org/licenses/by/4.0/), which permits unrestricted use, distribution, and reproduction in any medium, provided the original work, first published in Iproceedings, is properly cited. The complete bibliographic information, a link to the original publication on http://www.iproc.org/, as well as this copyright and license information must be included. 